\title{
Some Improvements to Total Degree Tests *
}

\author{
KATALIN FRIEDL $^{\dagger} \quad$ MADHU SUdAN $^{\ddagger}$
}

\begin{abstract}
A low-degree test is a collection of simple, local rules for checking the proximity of an arbitrary function to a lowdegree polynomial. Each rule depends on the function's values at a small number of places. If a function satisfies many rules then it is close to a low-degree polynomial. Low-degree tests play an important role in the development of probabilistically checkable proofs.

In this paper we present two improvements to the efficiency of low-degree tests. Our first improvement concerns the smallest field size over which a low-degree test can work. We show how to test that a function is a degree $d$ polynomial over prime fields of size only $d+2$.

Our second improvement shows a better efficiency of the low-degree test of [14] than previously known. We show concrete applications of this improvement via the notion of "locally checkable codes". This improvement translates into better tradeoffs on the size versus probe complexity of probabilistically checkable proofs than previously known.
\end{abstract}

\section{Introduction}

In this paper we consider functions mapping $m$ variables from some finite field $F$ to the same field. Let the dis-

\footnotetext{
* A version of this paper appeared in Proceedings of the 3rd Israel Symposium on Theory of Computing and Systems, Tel Aviv, Israel, January 4-7, 1995. This version corrects a few typographical errors.

$\dagger$ Computer and Automation Research Institute, Hungarian Academy of Sciences. e-mail: kati@ilab.sztaki.hu.. Most of this work was done while at the Department of Computer Science, University of Chicago. Supported in part by OTKA Grant 2581.

$\ddagger$ Research Division, IBM T.J. Watson Research Center, P.O. Box 218, Yorktown Heights, NY 10598, USA. e-mail: madhu@watson. ibm. com.
}

tance between two functions $f$ and $g$, denoted by $d(f, g)$ be $\operatorname{Pr}_{x \in F^{m}}[f(x) \neq g(x)]$. We use $\operatorname{deg}(f)$ to denote the total degree of $f$, and $\operatorname{deg}_{\max }(f)$ to denote the largest individual degree in any of the variables in $f$.

The low-degree testing problem for total degree (maximum degree) is defined as follows:

Definition 1 For parameters $d \in \mathcal{Z}^{+}$and $\delta, \epsilon \in \mathcal{R}^{+}, a$ low-degree tester is probabilistic oracle machine $\mathcal{T}$, that has access to a function $f: F^{m} \rightarrow F$ as an oracle, and behaves as follows:

- If $\operatorname{deg}(f) \leq d\left(\operatorname{deg}_{\max }(f) \leq d\right)$ then $T^{f}$ accepts.

- If for all total degree (maximum degree) d polynomials $g, d(f, g)>\epsilon$, then $T$ rejects with probability $\delta$.

The low-degree testing problem has been studied widely due to their relationship with probabilistically checkable (holographic) proofs and program checking. [5, 6, 9, 2, $10,13]$ study the case of testing the maximum degree and $[8,11,14,1]$ study the case of testing total degree ${ }^{1}$. Our improvements are to the latter family of testers. We start by describing their testers.

Definition 2 For points $x, h \in F^{m}$, the line through $x$ with offset $h$ is the set of points $l_{x, h}=\left\{l_{x, h}(t)=x+\right.$ $t \cdot h \mid t \in F\}$.

Definition 3 Given a function $f: F^{m} \rightarrow F$, a positive integer $d$ and points $x, h \in F^{m}$, the line polynomial $P_{x, h}^{(f, d)}: F \rightarrow F$ is a univariate polynomial of degree at most $d$ which satisfies $P_{x, h}^{(f, d)}(t)=f(x+t \cdot h)$ for the most $t$. Ties are broken arbitrarily.

The tester in [14] is effectively the following: "Pick $x, h$ randomly and uniformly from $F^{m}$ and $t$ randomly from $F$

\footnotetext{
${ }^{1}$ Some of the improvements in the former family have also affected the latter. In particular [1] obtain their improvement using the improved analysis of [2]. Similarly the work of [13] also affects the latter family of testers.
} 
and verify that $P_{x, h}^{(f, d)}(t)=f(x+t \cdot h)$." For our purposes it is not important how $P^{(f, d)}$ is computed by the tester. This will become clear in the context of our applications. The correctness of the tester is proved by the following kind of a statement.

Informal Statement: If $|F|$ is a sufficiently large function of $d$ and $\delta$ is a sufficiently small function of $d$, then given a function $f: F^{m} \rightarrow F$, if there exists a set of degree $d$ polynomials $\left\{P_{x, h}\right\}$ which satisfies

$$
\operatorname{Pr}_{x, h, t}\left[P_{x, h}(t) \neq f(x+t \cdot h)\right] \leq \delta
$$

then there exists a degree d polynomial $g: F^{m} \rightarrow F$ such that $d(f, g) \leq 2 \delta$.

The above statement does not specify the conditions on $|F|$ and $\delta$, and determining the exact conditions on these parameters turn out to be the interesting aspect in the analysis of low-degree tests. The improvements noted in this paper apply to these two parameters.

\subsection{Reducing Field Size}

The motivation for the following theorem is primarily one of curiosity. The smallest field size over which polynomials of a given total degree exhibit sufficient "redundancy" to, say, enable the application of the Schwartz-Zippel like theorems $[16,18]$, is when the field size is at least $d+2$. The low-degree tester of [11] uses sets of the same size, i.e., $d+2$, as elementary test sets. Their proof manages to show that in a certain sense (see Lemma 5) fields of size $d+2$ are sufficient to show some sort of robustness. However their proof falls short of showing low-degree tests that work over fields of size $d+2$ because of the lack of an "exact characterization" (in the sense of [15]). We complement their work by providing an exact characterization of low-degree polynomials, which shows that their tester is good for prime fields of size $d+2$, and improves the bound for non-prime fields as well. We give examples to show that our characterizations are essentially the best possible.

Lemma 4 If $q$, the order of $F$, and $p$, its characteristic, satisfy $q-q / p-1 \geq d$ and $g: F^{m} \rightarrow F$ satisfy

$$
P_{x, h}^{(g, d)}(t)=g(x+t \cdot h) \text { for all } x, h, t
$$

then $g$ is a degree d polynomial.

We use the above statement in combination with the following Lemma from [11, 15], to get Theorem 6.
Lemma $5([11,15])$ There exists a constant $c$ such that if $|F| \geq d+2$ and $\delta \leq \frac{1}{c(d+1)^{2}}$ and $P$ and $f$ satisfy

$$
\operatorname{Pr}_{x, h, t}\left[P_{x, h}(t) \neq f(x+t \cdot h)\right] \leq \delta
$$

then there exists a function $g: F^{m} \rightarrow F$ such that $d(f, g) \leq 2 \delta$ and $g$ satisfies

$$
P_{x, h}^{(g, d)}(t)=g(x+t \cdot h) \text { for all } x, h \in F^{m} \text { and } t \in F .
$$

Theorem 6 Let $q$ denote the order of the field $F$ and $p$ its characteristic. Then there exists a constant $c$ such that if $q-q / p-1 \geq d$ and $\delta \leq \frac{1}{c(d+1)^{2}}$ and $P$ and $f$ satisfy

$$
\operatorname{Pr}_{x, h, t}\left[P_{x, h}(t) \neq f(x+t \cdot h)\right] \leq \delta
$$

then there exists a degree d polynomial $g: F^{m} \rightarrow F$ such that $d(f, g) \leq 2 \delta$.

In Section 2 we also show that the requirement on $|F|$ is the tightest possible in the following sense: For all $p, q, d$ such that $d>q-q / p-1$, we show that there exist functions $g: F^{m} \rightarrow F$ and $P_{x, h}: F \rightarrow F$, such that $\operatorname{deg}\left(P_{x, h}\right) \leq d$ and for all $x, h \in F^{m}, t \in F, P_{x, h}(t)=g(x+t \cdot h)$, but $\operatorname{deg}(g)>d$.

\subsection{Improving the efficiency}

Improving the second of the two parameters in the statement of the Informal Statement is a task of greater significance. (Here an improvement would imply a larger value of $\delta$.) The result in [11] shows that the test works for $\delta \leq O\left(1 / d^{2}\right)$. The improvements in [14] and [1] yielded $\delta \leq O(1 / d)$ and $\delta \leq \delta_{0}$ for some $\delta_{0}>0$ respectively. The constant $\delta_{0}$ coming from the latter analysis is not described explicitly but the number appears to be fairly small. Here we show that the theorem works for any $\delta<1 / 8$. More precisely,

Theorem 7 For every $\epsilon>0$, there exist $c<\infty$ such that for all $d$, if $|F| \geq c d$ the following holds. Given a function $f: F^{m} \rightarrow F$ and degree d polynomials $\left\{P_{x, h}\right\}$ satisfying

$$
\operatorname{Pr}_{x, h, t}\left[P_{x, h}(t) \neq f(x+t \cdot h)\right]=\delta \leq 1 / 8-\epsilon
$$

there exists a degree d polynomial g such that $d(f, g) \leq 2 \delta$.

Remark: The bound on the field size in the above theorem is also better than that of [1] who are only able to show it for $|F| \geq d^{3}$. However, this improvement can already be inferred in the work of [13]. In fact, our analysis inherits this particular improvement from their analysis. 


\subsection{An Application}

The second theorem given above turns out to have some implication to the properties of probabilistically checkable proofs. In particular it implies that the proofs constructed in [1] have a much smaller probe complexity than shown earlier. It turns out that all the known construction of holographic proof inherits part of their properties from the properties of some underlying error-correcting code. It is easiest to describe the effect of our analysis in terms of the improvement in the properties of the codes created in $[1,15]$. The following definitions are from [15].

An $(k, n, d, a)$-code consists of an alphabet $\Sigma$ such that $\log |\Sigma|=a$ and a function $C: \Sigma^{k} \rightarrow \Sigma^{n}$, such that for any two strings $m, m^{\prime} \in \Sigma^{k}$, the (Hamming) distance between $C(m)$ and $C\left(m^{\prime}\right)$ is at least $d$. For applications to probabilistically checkable proofs, it is sufficient to consider codes restricted a small range of these parameters. We call these the good codes. Such codes need to have constant relative distance. The encoded message is allowed to be much larger than the original message size, as long as the final length is polynomially bounded.

Definition 8 (Good Code) $A$ family of codes $\left\{C_{i}\right\}$ with parameters $\left(k_{i}, n_{i}, d_{i}, a_{i}\right)$ is good if $k_{i} \rightarrow \infty, n_{i}$ is upper bounded by some polynomial in $k_{i}, d_{i} / n_{i}>0$ and $a_{i}=\operatorname{polylog}\left(k_{i}\right)$.

For the application to probabilistically checkable proofs, the primary question is the following: "Does the code admit very simple randomized error detection?". This notion is formalized in the next definition. Intuitively the definition says that the error-detection can be done by probing just $p$ letters of a word to get a confidence $\delta$ that it is close to some codeword.

Definition 9 For a positive integer $p$ and a positive real number $\delta$, an $(n, k, d, a)$-code $C$ over the alphabet $\Sigma$ is $(p, \delta)$-locally testable if the following exist

- A probability space $\Omega$ which can be efficiently sampled.

- Functions $q_{1}, q_{2}, \ldots, q_{p}: \Omega \rightarrow\{1, \ldots, n\}$.

- A boolean function $V: \Omega \times \Sigma^{p} \rightarrow\{0,1\}$.

with the property that for all $w \in \Sigma^{n}$, if

$$
\operatorname{Pr}_{r \in \Omega}\left[V\left(r, w_{q_{1}(r)}, \ldots, w_{q_{p}(r)}\right)=0\right]<\delta
$$

then there exists a (unique) string $m \in \Sigma^{k}$ such that $d(w, C(m))<d / 2$. Conversely, if $w=C(m)$ for some $m$, then $V\left(r, w_{q_{1}(r)}, \ldots, w_{q_{p}(r)}\right)=1$ for all $r \in \Omega$.
The codes of [6] for instance produce good codes which are (polylog $\left.\left(k_{i}\right), \Omega(1)\right)$-locally testable. The work of [2] implicitly describe a related code which achieves both $p, \frac{1}{\delta}=O(1)$, but requires very large alphabet sizes to get this - namely their code requires $a_{i}=k_{i}^{\epsilon}$. The significant improvement in [1] is to get good codes which have $p=2$, $\delta>0$ with $a_{i}=\operatorname{polylog}\left(k_{i}\right)$. (By applying a recursive technique introduced by [2] to this code they later manage to reduce $a$ to a constant as well.) The code used by [1] is the following (see also [15]):

Definition 10 (Polynomial-Line Codes) Let $c_{1}>1$ and $c_{2} \geq 1$ be parameters. The polynomial-line codes $\left\{L_{m}\right\}$ are chosen by letting $d=\Theta\left(m^{c_{1}}\right)$ and picking a finite field $F$ of size $\Theta\left(d^{c_{2}}\right)$. The code works over the alphabet $\Sigma=F^{d+1}$. The message consists of $\left(\begin{array}{c}m+d \\ d\end{array}\right)$ field elements (or $\left(\begin{array}{c}m+d \\ d\end{array}\right) /(d+1)$ letters from $\left.\Sigma\right)$ and is viewed as an $m$-variate degree d polynomial specified by its coefficients. Given a message polynomial $f$, the codeword consists of $\left\{P_{x, h}^{(f, d)}\right\}_{x, h \in F^{m}}$ where $P_{x, h}^{(f, d)}$ is the line polynomial for the line $l_{x, h}$ described by its $d+1$ coefficients. The code achieves $k_{m}=\left(\begin{array}{c}m+d \\ d\end{array}\right) /(d+1)$ and $n_{m}=|F|^{O\left(m^{2}\right)}$ over the alphabet $F^{d+1}$.

It is clear that for all constants $c_{1}$ and $c_{2}$ the PolynomialLine codes are good codes. [1] show that for all $c_{1}>1$ and $c_{2} \geq 3$ these codes give $(2, \delta>0)$-locally testable codes. [13] improve this to $c_{2} \geq 1$, without changing the $\delta$ in any significant way. Our analysis (Theorem 7) immediately yields that Polynomial-Line Codes are $(2,1 / 8-\epsilon)$ locally testable. It can be easily shown that no code can achieve $(2,1 / 2+\epsilon)$-local testability. Thus in this case our results come close to optimality.

Connection with proof checking Lastly we describe a very informal manner the way in which this affects the construction of probabilistically checkable proofs. We assume that the reader of this subsection is familiar with the notion of probabilistically checkable proofs (PCPs) as defined in [2] (see, for instance, [3] for a survey). In particular we discuss the probe complexity of proofs and the sizes of probabilistically checkable proofs.

As mentioned earlier every holographic proof ends up inheriting part of its properties on some underlying locallytestable code. In order to test that a given proof is valid one ends up testing that the proof corresponds to a valid codeword. This effectively implies that to obtain a fixed degree of confidence, one has to look at $O(p / \delta)$ letters in the proof. Thus the probe complexity of a PCP seems to be inherently dependent on the ratio of $p$ and $\delta$.

However, the relationship between $p / \delta$ and the probe com- 
plexity of PCP turns out to be not so simple. [7] manage to reduce the probe complexity of a PCP to about 24 bits to get a confidence of $1 / 2$ (from some unknown number estimated to be around $10^{4}$ in [1]) without improving the analysis of low-degree tests! How do they obtain this reduction? It turns out that this reduction is obtained by exploding the proof size to the order of $n^{10^{4}}$ (from some smaller polynomial of size about $n^{12}$ in [1]). But by incorporating the analysis from this paper into the analysis of PCP one can obtain better bounds on the probe complexity of proof systems. The verifier we construct probes a proof at most 165 bits (as opposed to the $10^{4}$ of $[1,13]$ ) while increasing the proof size to only $n^{2+\epsilon}$ (to be contrasted with the $n^{10^{4}}$ in [7]). (We point out that the improvement relies fairly heavily on the techniques developed in [7] and [13], as well as those of [2] and [1].)

\section{Characterizing the Total Degree of Polynomials}

Let $F=F_{q}$ be a finite field of order $q=p^{s}$ where $p$ is its characteristic.

Theorem 11 Let $g: F^{m} \rightarrow F$ be a function which satisfies

$$
\forall x, h \in F^{m}, t \in F \quad P_{x, h}^{(g, d)}(t)=g(x+t \cdot h) .
$$

Then if $q-q / p-1 \geq d$, then $g$ is a polynomial of degree at most $d$.

Remark. The inequality $q-q / p-1 \geq d$ in the Theorem cannot be weakened for any $q$. Indeed, for any $d$ such that $q-q / p-1<d<q$, consider the bivariate function $g\left(x_{1}, x_{2}\right)=\left(x_{1}^{(p-1)} x_{2}\right)^{q / p}$. For every pair $x, h$, the univariate function $P_{x, h}$ given by $P_{x, h}(t)=g(x+t \cdot h)$. Each term in this univariate polynomial has degree at most $(p+1) q / p=q+q / p$ and each exponent is divisible by $q / p$. As a function we have $t^{q}=t$, and thus $\operatorname{deg}\left(P_{x, h}\right)$ is at most $q-q / p$. Thus we have $P_{x, h}^{(g, d)} \equiv P_{x, h}$. On the other hand the total degree of $g$ is $q>d$.

For the proof of Theorem 11, we first prove a lemma about the behavior of the binomial coefficients modulo $p$.

Lemma 12 Let $0<r \leq n \leq p^{s}-1$. If $r=k p^{s-1}$ then $\left(\begin{array}{l}n \\ r\end{array}\right)$ is not divisible by $p$.

Proof: For any positive integer $l$, the largest power of $p$ that divides $l !$ is $\lfloor l / p\rfloor+\left\lfloor l / p^{2}\right\rfloor+\left\lfloor l / p^{3}\right\rfloor+\cdots$. But for $r=k p^{s-1}$, the identity $\left\lfloor n / p^{i}\right\rfloor=\left\lfloor r / p^{i}\right\rfloor+\lfloor(n-$ $\left.r) / p^{i}\right\rfloor$ holds. Thus the largest power of $p$ that divides $n$ ! is $\sum_{i=1}^{\infty}\left\lfloor n / p^{i}\right\rfloor=\sum_{i=1}^{\infty}\left(\left\lfloor r / p^{i}\right\rfloor+\left\lfloor(n-r) / p^{i}\right\rfloor\right)$. Therefore $n$ ! and $r !(n-r)$ ! are divisible by exactly the same power of $p$.

Proof of Theorem 11: Assume for the sake of contradiction that the assertion of the theorem is false. Let $m$ be the smallest positive integer for which the following holds:

$$
\begin{aligned}
\exists g: & F^{m} \rightarrow F \text { s.t. } \forall x, h \in F^{m}, t \in F \\
& P_{x, h}^{(g, d)}(t)=g(x+t \cdot h) \text { but } \operatorname{deg}(g)>d .
\end{aligned}
$$

Express $g$ in the form:

$$
g\left(x_{1}, \ldots, x_{m}\right)=\sum_{i_{1}=0}^{q-1} \cdots \sum_{i_{m}=0}^{q-1} \alpha_{i_{1}, \ldots, i_{m}} x_{1}^{i_{1}} \cdots x_{m}^{i_{m}} .
$$

(Notice that there exists $\alpha$ 's such that the above is true, and these are unique.) Since $g$ is not a degree $d$ polynomial, there exist $l$ and $i_{1}, \ldots, i_{m}$ such that $\sum_{j=1}^{m} i_{j}=l>d$ and $\alpha_{i_{1}, \ldots, i_{m}} \neq 0$. Let $l$ be the largest integer with this property. We consider the following cases:

Case: $\sum_{j=1}^{m-1} i_{j}>d$ : We show that this contradicts the assumption that $m$ is the smallest integer for which (1) holds. For $a_{m} \in F$, let $g_{a_{m}}: F^{m} \rightarrow F$ be given by $g_{a_{m}}\left(x_{1}, \ldots, x_{m-1}\right)=g\left(x_{1}, \ldots, x_{m-1}, a_{m}\right)$. Notice first that $g_{a_{m}}$ satisfies $g_{a_{m}}(x+t \cdot h)=P_{x, h}^{\left(g_{a_{m}}, d\right)}(t)$ for all $x, h \in F^{m-1}$ and $t \in F$. This follows from the fact that $P_{x, h}^{\left(g_{a_{m}}, d\right)}(\cdot)=P_{x^{\prime}, h^{\prime}}^{(g, d)}(\cdot)$ for $x^{\prime}=<x, a_{m}>$ and $h^{\prime}=<h, 0>$. We now show that there exists $a_{m}$ such that $\operatorname{deg}\left(g_{a_{m}}\right)>d$. Observe that the coefficient for $x_{1}^{i_{1}} \cdots x_{m-1}^{i_{m-1}}$ is $\sum_{i=0}^{q-1} \alpha_{i_{1}, \ldots, i_{m-1}, i} a_{m}^{i}$. This summation is a non-zero polynomial in $a_{m}$ of degree less than $q$. Thus there must exist a point $a_{m}$ where the summation is non-zero. This gives us $a_{m}$ such that $g_{a_{m}}$, a function of $m-1$ variables, satisfies (1). As promised, this violated the minimality of $m$.

Case: $\sum_{j=2}^{m} i_{j}>d:$ Similar to above.

Case: $l=\sum_{j=1}^{m} i_{j}<q$ : For $a_{1}, \ldots, a_{m} \in F$, let $g_{a_{1}, \ldots, a_{m}}: F \rightarrow F$ be given by $g_{a_{1}, \ldots, a_{m}}(t)=$ $g\left(a_{1} t, \ldots, a_{m} t\right)$. The coefficient of $t^{l}$ in $g_{a_{1}, \ldots, a_{m}}$ is given by

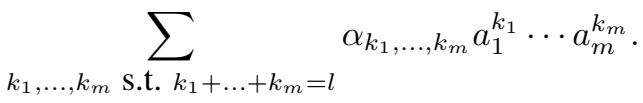


Since this expression is a polynomial in the $a_{j}$ 's of degree less than $q$ and is not identically zero, there exist $a_{1}, \ldots, a_{m}$ for which the coefficient of $t^{l}$ in $g_{a_{1}, \ldots, a_{m}}(t)$ is non-zero. But for $x=0$ and $a=<$ $a_{1}, \ldots, a_{m}>$, we find that $P_{x, a}^{(g, d)} \equiv g_{a_{1}, \ldots, a_{m}}$. and the fact that $g_{a_{1}, \ldots, a_{m}}$ is not a polynomial of degree $d$ contradicts the conditions guaranteed in (1).

Case: None of the above: In this case we have $\sum_{j=1}^{m-1} i_{j} \leq d, i_{m} \leq \sum_{j=2}^{m} i_{j} \leq d$ and $l \geq$ $q$. Here we consider the function $g_{a_{1}, \ldots, a_{m-1}, b}(t)=$ $g\left(a_{1} t, \ldots, a_{m-1} t, b+t\right)$ and show that for some choice of $a_{1}, \ldots, a_{m-1}$ and $b$, the coefficient of $t^{r}$ in $g_{a_{1}, \ldots, a_{m-1}, b}$ is non-zero, for some $r$ in the range $[d+1, q]$, of the form $n p^{s-1}+\sum_{j=1}^{m-1} i_{j}$. Such a choice for $r$ exists since the range $[d+1, q]$ contains at least $q-d-1 \geq q / p=p^{s-1}$ elements.

We start with the observation that the coefficient of $t^{r}$ in the function $g_{a_{1}, \ldots, a_{m-1}, b}(t)$ is the same as the coefficient of $t^{r}$ in the formal power series expansion of $g$ with the formal substitutions $x_{i}=a_{i} t$ and $x_{m}=$ $b+t$. This is true because the formal power series contains terms of degree at most $l$ and $l$ satisfies the condition: $q+r>l$. (Since $l=i_{m}+\sum_{j=1}^{m-1} i_{j} \leq$ $i_{m}+r \leq d+r<r+q$.)

The coefficient of $t^{r}$ in the formal expansion is

$$
\sum\left(\begin{array}{c}
k_{m} \\
r-\sum_{i=1}^{m-1} k_{i}
\end{array}\right) \alpha_{k_{1}, \ldots, k_{m}} \prod_{i=1}^{m-1} a_{i}^{k_{i}} b^{\sum_{i=1}^{m} k_{i}-r}
$$

where the summation ranges over all choices of $k_{1}, \ldots, k_{m}$ such that $r \leq \sum_{j=1}^{m} k_{j} \leq l$. Thus coefficient is a polynomial in $a_{j}$ 's and $b$ of degree at most $q$ in each variable. Moreover for $k_{j}=i_{j}$, the term $\alpha_{k_{1}, \ldots, k_{m}}$ is non-zero and the term $\left(\begin{array}{c}k_{m} \\ r-k_{1}-\cdots-k_{m-1}\end{array}\right)$ simplifies to $\left(\begin{array}{c}i_{m} \\ n p^{s-1}\end{array}\right)$ which is also non-zero (by Lemma 12). Thus the coefficient of $t^{r}$ is a non-zero polynomial of maximum degree at most $q$. Hence there exists a choice of $a_{1}, \ldots, a_{m-1}$ and $b$ such that the coefficient of $t^{r}$ is non-zero modulo $p$.

We now obtain the contradiction in the usual way. We observe that $g_{a_{1}, \ldots, a_{m-1}, b} \equiv P_{x, h}^{(g, d)}$ for $x=<$ $0, \ldots, 0, b>$ and $h=<a_{1}, \ldots, a_{m-1}, 1>$. Thus $g_{a_{1}, \ldots, a_{m-1}, b}$ should be a polynomial of degree at most $d$, contradicting the fact that the coefficient of $t^{r}$ is non-zero.

\section{Efficiency of the Lines test}

The main theorem of this section is motivated by the following tester: The tester $\mathcal{T}$ is provided access to an oracle for $f: F^{m} \rightarrow F$ and an augmenting oracle $O: F^{2 m} \rightarrow$ $F^{d+1}$. The augmenting oracle takes as input the description of a line by the pair $x, h \in F^{m}$ and provides the coefficients of the "line polynomial" $P_{x, h}$. The effect of Theorem 13 is to show that the tester behaves as follows:

- If $f$ is a degree $d$ polynomial then there exists an $O$ such that $\mathcal{T}^{f, O}$ always accepts.

- If $d(f, g) \geq 1 / 4$ for every degree $d$ polynomial $g$, then for every oracle $O: F^{2 m} \rightarrow F^{d+1}, \mathcal{T}^{f, O}$ rejects with probability at least $1 / 8-\epsilon$.

- $\mathcal{T}$ makes exactly one call to each oracle (i.e., $f$ and $O)$.

The consequences of this theorem are summarized in Section 4.

Theorem 13 For every $\epsilon>0$, there exists $c<\infty$ such that for all $d \in \mathcal{Z}^{+}$if $|F| \geq c d$ then the following holds. Given a function $f: F^{m} \rightarrow F$ and degree d polynomials $\left\{P_{x, h}\right\}$ such that:

$$
\operatorname{Pr}_{x, h, t}\left[P_{x, h}(t) \neq f(x+t \cdot h)\right]=\delta \leq 1 / 8-\epsilon
$$

there exists a degree d polynomial $g$ such that $d(f, g) \leq 2 \delta$.

Our proof is based on the proof in [15] and borrows various ingredients from their technique. However our analysis seems to be simplify certain aspects of their proof by introducing an inductive analysis to their proof. The improvement in the value of $\delta$ is obtained by very careful sampling of the underlying space and the application of pairwise independent analysis to their space. The use of pairwise independent analysis in low-degree testing seems to be new.

In what follows we fix an $\epsilon>0$. We assume that $c \rightarrow$ $\infty$. Thus whenever the notation $\alpha=o(1)$ is used in what follows, it implies that $\alpha \rightarrow 0$ as $c \rightarrow \infty$.

We start with a couple of definitions. Given a function $f$ : $F^{m} \rightarrow F$, let $\delta_{f}$ be defined as

$$
\delta_{f}=\underset{x, h}{\operatorname{Pr}}\left[f(x) \neq P_{x, h}^{(f, d)}(0)\right]
$$

and let $\operatorname{Corr}_{f}: F^{m} \rightarrow F$ be the function defined by

$$
\operatorname{Corr}_{f}(x)=\text { plurality }_{h}\left\{P_{x, h}^{(f)}(0)\right\}^{2} .
$$

\footnotetext{
${ }^{2}$ The plurality of a multiset is the most commonly occurring element in the multiset. We use the word plurality as opposed to majority since
} 
We start with a few basic facts about $\delta_{f}$ and $\operatorname{Corr}_{f}$.

Fact 14 For any function $f: F^{m} \rightarrow F$, and degree $d$ polynomials $\left\{P_{x, h}: F \rightarrow F\right\}_{x, h \in F^{m}}$,

$$
\operatorname{Pr}_{x, h, t}\left[f(x+t \cdot h) \neq P_{x, h}(t)\right] \geq \delta_{f} .
$$

The above fact follows directly from the fact that for each $x, h P_{x, h}^{f, d}$ minimizes (over random $t$ ) the probability that $f(x+t \cdot h) \neq P_{x, h}(t)$.

Lemma $15([11]) d\left(f\right.$, Corr $\left._{f}\right) \leq 2 \delta_{f}$.

Lemma 16 ([11]) For all $\beta>0$, if $g$ is a degree d polynomial such that $d(f, g)<1 / 4-\beta$, then Corr $_{f} \equiv g$.

We need a slightly stronger version of the above lemma for our purposes which we prove next.

Lemma 17 For all $\beta>0$, if $g: F^{m} \rightarrow F$ is a degree $d$ polynomial such that $d(f, g)<1 / 2-\beta$ then $d\left(\operatorname{Corr}_{f}, g\right)=$ $o(1)$.

Proof: Consider randomly chosen $x, h \in F^{m}$ and the line $l_{x, h}$. Notice that this line represents a pairwise independent collection of points from $F^{m}$. Thus with probability $1-\alpha$, where $\alpha=o(1)$, the number of points, $y$, on $l_{x, h}$ such that $f(y) \neq g(y)$ is less than $1 / 2-\epsilon / 2$ and in such cases $P_{x, h}^{(f, d)} \equiv P_{x, h}^{(g, d)}$.

Now

consider the set $B=\left\{x \mid P_{x, h}^{(f, d)} \not \equiv P_{x, h}^{(g, d)}\right.$ for a majority of $h \in$ $\left.F^{m}\right\}$. Based on the above argument notice that the fraction $|B| /|F|^{m}$ is at most $2 \alpha=o(1)$. But for $x \notin B$, we have $\operatorname{Corr}_{f}(x)=g(x)$.

The main lemma we prove is the following:

Lemma $18 \forall f: F^{m} \rightarrow F$ s.t. $|F|>16 / \epsilon^{2}$ and $\delta_{f} \leq$ $1 / 8-\epsilon, \delta_{\text {Corr }_{f}}<\delta_{f}$.

We defer the proof to the next subsection. We first show why this suffices.

Proof of Theorem 13: We prove this theorem by induction of $\delta$. (Observe that since we are talking of functions over finite domains, $\delta$ can only take finitely many values.) Say the theorem is true for functions $f,\left\{P_{x, h}\right\}$ with

$$
\operatorname{Pr}_{x, h, t}\left[P_{x, h}(t) \neq f(x+t \cdot h)\right]<\delta .
$$

the latter could also be used to point to the (unique) element that occurs with frequency more than half.
Now consider functions $f,\left\{P_{x, h}\right\}$ with

$$
\operatorname{Pr}_{x, h, t}\left[P_{x, h}(t) \neq f(x+t \cdot h)\right]=\delta .
$$

For such a $f$ consider the function Corr $f$. By Lemma 18,

$$
\underset{x, h, t}{\operatorname{Pr}}\left[P_{x, h}^{\left(\operatorname{Corr}_{f}, d\right)}(t) \neq \operatorname{Corr}_{f}(x+t \cdot h)\right]=\delta_{\operatorname{Corr}_{f}}<\delta_{f} .
$$

By induction there exists a degree $d$ polynomial $g$ such that $d\left(\operatorname{Corr}_{f}, g\right) \leq 2 \delta_{\text {Corr }_{f}}<2 \delta_{f}$. By Lemma 15 $d\left(f\right.$, Corr $\left._{f}\right) \leq 2 \delta_{f}$. Thus $d(f, g) \leq 4 \delta_{f} \leq 4 \delta \leq 1 / 2-4 \epsilon$. By Lemma $17 d\left(\operatorname{Corr}_{f}, g\right)=o(1)$. This in turn implies that $d(f, g) \leq 1 / 4-\epsilon+o(1)$. By Lemma 16 we now conclude that $\operatorname{Corr}_{f}=g$ implying that $d(f, g) \leq 2 \delta_{f}$.

\subsection{Proof of Main Lemma}

The proof of Lemma 18 relies on a minor strengthening of the following lemma due to [13], which in turn improves upon a similar lemma in [2].

Lemma 19 ([13]) For any $\epsilon>0$, if $r_{i}$ and $c_{j}$ are families of degree d polynomials such that

$$
\operatorname{Pr}_{i, j \in F}\left[r_{i}(j) \neq c_{j}(i)\right] \leq 1 / 4-\epsilon,
$$

then there exists a bivariate polynomial $Q$ of degree $d$ in each variable such that

$$
\operatorname{Pr}_{i, j \in F}\left[r_{i}(j) \neq Q(i, j) \text { or } c_{j}(i) \neq Q(i, j)\right] \leq 1 / 2-\epsilon .
$$

We first strengthen the conclusion obtained above slightly.

Lemma 20 Let $\epsilon \geq d /|F|$ and Let $r_{i}$ and $c_{j}$ be families of degree d polynomials such that

$$
\operatorname{Pr}_{i, j \in F}\left[r_{i}(j) \neq c_{j}(i)\right] \leq 1 / 4-\epsilon .
$$

Then there exists a bivariate polynomial $Q$ of degree $d$ in each variable such that

$$
\begin{gathered}
\operatorname{Pr}_{i \in F}\left[r_{i}(\cdot) \neq Q(i, \cdot)\right] \leq 1 / 4 \\
\text { and } \operatorname{Pr}_{j \in F}\left[c_{j}(\cdot) \neq Q(\cdot, j)\right] \leq 1 / 4 .
\end{gathered}
$$

Proof: This lemma follows in a straightforward manner from Lemma 19. Let $Q$ be the bivariate polynomial guaranteed by Lemma 19. We define the bad rows and bad columns as follows. Let

$$
B_{\text {row }}=\left\{i \in F \mid r_{i}(\cdot) \neq Q(i, \cdot)\right\} \text { and let } x=\left|B_{\text {row }}\right| /|F| \text {. }
$$


Similarly let

$$
B_{\mathrm{col}}=\left\{j \in F \mid c_{j}(\cdot) \neq Q(\cdot, j)\right\} \text { and let } y=\left|B_{\mathrm{col}}\right| /|F| .
$$

We count the number of points in $B_{\text {row }} \times\left(F-B_{\text {col }}\right)$ which satisfy $r_{i}(j) \neq c_{j}(i)$. For each bad row $i$, there are at most $d$ points for which $r_{i}(j)=Q(i, j)$. All the remaining points must lie on a bad column or must satisfy $r_{i}(j) \neq$ $c_{j}(i)$. Thus the fraction of violations in any bad row (from the good columns) is at least $(1-d /|F|-y)$. Similarly we count the violations in bad columns and good rows and summing all theses violations we get:

$$
\begin{aligned}
1 / 4- & \epsilon \\
\geq & \operatorname{Pr}_{i, j}\left[r_{i}(j) \neq c_{j}(i)\right] \\
\geq & \left(\operatorname{Pr}_{i}\left[i \in B_{\text {row }}\right]\right. \\
& \left.* \operatorname{Pr}_{j}\left[j \notin B_{\text {col }} \text { and } c_{j}(i) \neq r_{i}(j) \mid i \in B_{\text {row }}\right]\right) \\
& +\left(\operatorname{Pr}_{j}\left[j \in B_{\text {col }}\right]\right. \\
& \left.* \operatorname{Pr}_{i}\left[i \notin B_{\text {row }} \text { and } c_{j}(i) \neq r_{i}(j) \mid j \in B_{\text {col }}\right]\right) \\
\geq & x\left(1-y-\frac{d}{|F|}\right)+y\left(1-x-\frac{d}{|F|}\right) .
\end{aligned}
$$

We now use the fact that $x, y \leq 1 / 2$ and that $\epsilon \geq d /|F|$, to reduce the above to $x \leq 1 / 4$ and $y \leq 1 / 4$.

We are now almost ready to prove Lemma 18 . We first prove a variant and then show how it implies the final result.

Lemma 21 If $\delta_{f} \leq 1 / 8-\epsilon$, then for $x, h_{1}, h_{2}$ chosen uniformly at random from $F^{m}$,

$$
\underset{x, h_{1}, h_{2}}{\operatorname{Pr}}\left[P_{x, h_{1}}^{(f, d)}(0) \neq P_{x, h_{2}}^{(f, d)}(0)\right] \leq 4 \alpha \delta_{f} \text { where } \alpha=\frac{4}{\epsilon^{2}|F|} .
$$

Proof: Pick $x, h_{1}, h_{2}, h_{3}$ at random from $F^{m}$ and consider the set of points $\left\{x+i h_{1}+j h_{2}+i j h_{3} \mid i, j \in F\right\}$. We partition this set in two ways - by "rows" and by "columns" as follows. For $i \in F$ let row $i=\left\{x+i h_{1}+j h_{2}+i j h_{3} \mid j \in\right.$ $F\}$. Similarly for $j \in F$ let $\operatorname{col}_{j}=\left\{x+i h_{1}+j h_{2}+\right.$ $\left.i j h_{3} \mid i \in F\right\}$. Notice that each row and column is a line from the space $F^{m}$. We first observe that these are actually random lines (Here we call the distribution of lines picked by choosing a line $l_{x, h}$ by picking $x, h \in F^{m}$ uniformly and randomly, to be the uniform distribution over lines.)

Claim 22 For $i_{1} \neq i_{2} \in F$, the rows row $i_{1}$ and row $_{i_{2}}$ are independently and uniformly distributed over lines in $F^{m}$. (Similarly for the columns.)

Let $m(i, j)=f\left(x+i h_{1}+j h_{2}+i j h_{3}\right)$. Further let $r_{i}(\cdot)=$

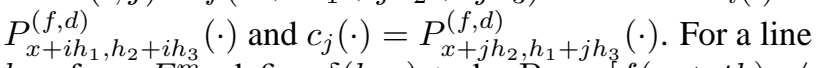
$l_{x, h}$ from $F^{m}$, define $\delta\left(l_{x, h}\right)$ to be $\operatorname{Pr}_{t \in F}[f(x+t h) \neq$ $\left.P_{x, h}^{(f, d)}(t)\right]$. Notice that $E_{x, h}\left[\delta\left(l_{x, h}\right)\right]=\delta_{f}$. The pairwise independence of the lines implies that the collection of real numbers $\left\{\delta\left(\operatorname{row}_{i}\right)\right\}_{i \in F}$ is a pairwise independent collection of variables taking values from $[0,1]$ with expectation $\delta_{f}$. The second moment method thus allows us to estimate the mean of this sample and shows that:

$$
\begin{gathered}
\operatorname{Pr}_{x, h_{1}, h_{2}, h_{3}}\left[\sum_{i} \delta\left(\operatorname{row}_{i}\right) /|F| \geq 1 / 8-\epsilon / 2\right] \\
\leq \alpha \delta_{f}\left(1-\delta_{f}\right) \text { where } \alpha=\frac{4}{\epsilon^{2}|F|} .
\end{gathered}
$$

A similar analysis applied to the columns yields:

$$
\begin{gathered}
\operatorname{Pr}_{x, h_{1}, h_{2}, h_{3}}\left[\sum_{j} \delta\left(\operatorname{col}_{j}\right) /|F| \geq 1 / 8-\epsilon / 2\right] \\
\leq \alpha \delta_{f}\left(1-\delta_{f}\right) \text { where } \alpha=\frac{4}{\epsilon^{2}|F|} .
\end{gathered}
$$

By combining (2) and (3) yields that with probability all but at most $2 \alpha \delta_{f}$ over four tuples $\left(x, h_{1}, h_{2}, h_{3}\right)$ we have, $\operatorname{Pr}_{i, j \in F}\left[r_{i}(j) \neq c_{j}(i)\right] \leq 1 / 4-\epsilon$. This allows us to apply Lemma 20 to claim that for at least $3 / 4$ fraction of the $i$ 's, $r_{i}(\cdot) \equiv Q(i, \cdot)$ (and similarly for the columns).

Once again, we use pairwise independence to show that

$$
\begin{aligned}
& \operatorname{Pr}_{x, h_{1}, h_{2}, h_{3}}\left[\left\{i \in F \mid r_{i}(0) \neq m(i, 0)\right\}\right. \\
& \quad \geq(1 / 8-\epsilon / 2)|F|] \\
& \leq \alpha \delta_{f}\left(1-\delta_{f}\right) \text { where } \alpha=\frac{4}{\epsilon^{2}|F|} . \\
& \operatorname{Pr}_{x, h_{1}, h_{2}, h_{3}}\left[\left\{j \in F \mid c_{j}(0) \neq m(0, j)\right\}\right. \\
& \quad \geq(1 / 8-\epsilon / 2)|F|] \\
& \leq \quad \alpha \delta_{f}\left(1-\delta_{f}\right) \text { where } \alpha=\frac{4}{\epsilon^{2}|F|} .
\end{aligned}
$$

Thus we now see that with probability at least $1-4 \alpha \delta_{f}$ all the events in (2), (3), (4) and (5) hold. In this case $m(i, 0)=Q(i, 0)$ for at least $3 / 4-1 / 8+\epsilon$ fraction of $i \in F$, which implies that $c_{0}(\cdot)=Q(\cdot, 0)$. Thus we have $P_{x, h_{2}}^{(f)}(\cdot)=c_{0}(\cdot)=Q(\cdot, 0)$. Similarly $P_{x, h_{1}}^{(f)}(\cdot)=Q(0, \cdot)$. Thus $P_{x, h_{2}}^{(f)}(0)=P_{x, h_{1}}^{(f)}(0)=Q(0,0)$.

Proof of Lemma 18: We start with the following observation:

$$
\begin{aligned}
\forall x, \quad & \quad \operatorname{Pr}\left[\text { plurality }_{h_{1}}\left\{P_{x, h_{1}}^{(f, d)}(0)\right\} \neq P_{x, h_{2}}^{(f, d)}(0)\right] \\
\leq & \operatorname{Pr}_{h_{1}, h_{2}}\left[P_{x, h_{1}}^{(f, d)}(0) \neq P_{x, h_{2}}^{(f, d)}(0)\right] .
\end{aligned}
$$


We prove the above by running two different probabilistic experiments. Say, a bag has a number of colored balls, with the distribution of the number of balls of each color being known. In the first game we nominate a color and then pick a random ball and we lose if the color of the randomly chosen ball is different from the nominated one. In the second game we pick two balls (with replacement) at random from the bag and lose if the balls have different colors. It is clear that in the first game the best choice is to deterministically pick the most often occuring color in the bag, while the second game corresponds to a mixed strategy for nominating the color in the first game. Thus we are no more likely to lose in the first game than in the second. The inequality above represents this analysis, with the $h$ 's corresponding to the balls and $P_{x, h}^{(f, d)}(0)$ 's corresponding to their colors.

We now use the inequality above as follows:

$$
\begin{gathered}
E_{x}\left[\underset{h_{2}}{\operatorname{Pr}}\left[\begin{array}{r}
\operatorname{Corr}_{f}(x)=\text { plurality }_{h_{1}}\left\{P_{x, h_{1}}^{(f, d)}(0)\right\} \\
\neq P_{x, h_{2}}^{(f, d)}(0)
\end{array}\right]\right] \\
\leq E_{x}\left[\underset{h_{1}, h_{2}}{\operatorname{Pr}}\left[P_{x, h_{1}}^{(f, d)}(0) \neq P_{x, h_{2}}^{(f, d)}(0)\right]\right]
\end{gathered}
$$

In turn this implies

$$
\begin{aligned}
\delta_{\text {Corr }_{f}} & =\operatorname{Pr}_{x, h_{2}}\left[\operatorname{Corr}_{f}(x) \neq P_{x, h_{2}}^{(f, d)}(0)\right] \\
& \leq \operatorname{Pr}_{x, h_{1}, h_{2}}\left[P_{x, h_{1}}^{(f, d)}(0) \neq P_{x, h_{2}}^{(f, d)}(0)\right] .
\end{aligned}
$$

By Lemma 21 the last quantity above is bounded by $4 \alpha \delta_{f}$. Thus if we choose $|F|$ to be sufficiently large (strictly greater than $\left(16 / \epsilon^{2}\right)$ ) then we get the conclusion $\delta_{\text {Corr }_{f}}<$ $\delta_{f}$.

\section{Conclusions}

Here we list the two main consequences of Theorem 13. The first is a straightforward corollary of the efficiency of the lines test and talks about the local testability property of the Polynomial-Line Codes (see Definition 10).

Theorem 23 The Polynomial-Line Codes are $(2,1 / 8-\epsilon)$ locally testable.

By applying Theorem 13 to the task of constructing efficient probabilistic verifiers, we get small "transparent" proofs with low query complexity. The transparent proofs so obtained are only slightly super-quadratic $\left(n^{2+\epsilon}\right.$-sized where $n$ is the size of traditional proof) in the length of the traditional proofs and the verifier probes them in at most 165 bits and always accepts correct proofs, while rejecting incorrect theorems with probability $1 / 2$. To be able to lay out precise bounds on the size of the proof, one needs to be careful about the model of computing used to define the size of a proof. The model we use here is the same as that used by [13]. In fact our verifier uses theirs as a black box and then builds upon it. In addition to the use of such size-efficient proof systems our construction also use many ingredients from the query-efficient proofs of [7]. The recursion mechanism of [2] plays a central role in the combination of the various proof systems used here. The final ingredient in the proof system is the randomnessefficient parallelization protocol of [1] (which is where the efficiency of the tester of [14] plays a role). Details of the construction will be available in the full paper.

Last we would also like to mention two interesting questions that may be raised about locally checkable codes.

1. Does there exist a family of good $(2,1 / 2)$ locallycheckable codes?

2. Does there exist such a family of codes with constant alphabet size?

\section{Acknowledgments}

We would like to thank Laci Babai and Oded Goldreich for their valuable comments. We also thank Steven Phillips for providing us with a copy of the manuscript [12].

\section{References}

[1] S. Arora, C. Lund, R. Motwani, M. Sudan, and M. Szegedy. Proof verification and the intractability of approximation problems. In Proceedings of the 33rd IEEE Symposium on Foundations of Computer Science, pages 14-23, 1992.

[2] S. Arora and S. Safra. Probabilistic checking of proofs: A new characterization of NP. In Proceedings of the 33rd Annual IEEE Symposium of the Foundations of Computer Science, pages 2-13, 1992.

[3] L. Babai. Transparent (holographic) proofs. SpringerVerlag Lecture Notes on Computer Science, 10th Annual Symposium on Theoretical Aspects of Computer Science, 665:525-533, 1993.

[4] L. Babai and K. Friedl. On slightly superlinear transparent proofs. Tech. Report CS 93-13, Department of Computer Science, University of Chicago, 1993. 
[5] L. Babai, L. Fortnow, and C. Lund. Nondeterministic exponential time has two-prover interactive protocols. Computational Complexity, 1 (1991), 3-40.

[6] L. Babai, L. Fortnow, L.A. Levin, and M. Szegedy. Checking computations in polylogarithmic time. In Proceedings of the 23rd STOC, pages 21-31, 1991.

[7] M. Bellare, S. Goldwasser, C. Lund, and A. Russell. Efficient probabilistically checkable proofs. In Proceedings of the 25th Annual ACM Symposium on Theory of Computing, pages 294-304, 1993.

[8] M. Blum, M. Luby, and R. Rubinfeld. Selftesting/correcting with applications to numerical problems. In Proceedings of the 22nd Annual ACM Symposium on Theory of Computing, pages 73-83, 1990.

[9] U. Feige, S. Goldwasser, L. Lovász, S. Safra, and M. Szegedy. Approximating clique is almost NPcomplete. In Proceedings of the 32nd IEEE Symposium on Foundations of Computer Science, pages 212, 1991.

[10] K. Friedl, Zs. Hátsági, and A. Shen. Low-degree tests. In Proceedings of the 5th ACM-SIAM Symposium on Discrete Algorithms, pages 57-64, 1994.

[11] P. Gemmell, R. Lipton, R. Rubinfeld, M. Sudan, and A. Wigderson. Self-testing/correcting for polynomials and for approximate functions. In Proceedings of the 23rd Annual ACM Symposium on Theory of Computing, pages 32-42, 1991.

[12] S. Phillips and S. Safra. Efficient Probabilistic Verification. Manuscript, 1992.

[13] A. Polishchuk and D. Spielman. Nearly-linear size holographic proofs. In Proceedings of the 26th Annual ACM Symposium on the Theory of Computing, pages 194-203.

[14] R. Rubinfeld and M. Sudan. Testing polynomial functions efficiently and over rational domains. In Proceedings of the 3rd Annual ACM-SIAM Symposium on Discrete Algorithms, pages 23-43, 1992.

[15] R. Rubinfeld and M. Sudan. Robust characterizations of polynomials with applications to program testing. Technical Report RC 19156, IBM Research Division, T. J. Watson Research Center, Yorktown Heights, NY 10598, September 1993.

[16] J.T. Schwartz. Fast probabilistic algorithms for verification of polynomial identities. Journal of the ACM, v. $27,701-717,1980$.
[17] M. Sudan. Efficient Checking of Polynomials and Proofs and the Hardness of Approximation Problems. PhD thesis, University of California at Berkeley, Berkeley, CA 94720, 1992.

[18] R. Zippel. Probabilistic algorithms for sparse polynomials. EUROSAM '79, Lecture Notes in Computer Science, 72:216-226, 1979. 